\title{
Control Design for Soft Robots based on Reduced Order Model
}

\author{
Maxime Thieffry ${ }^{1,2}$, Alexandre Kruszewski ${ }^{2}$, Christian Duriez ${ }^{2}$, and Thierry-Marie Guerra ${ }^{1}$
}

\begin{abstract}
Inspired by nature, soft robots promise disruptive advances in robotics. Soft robots are naturally compliant and exhibit nonlinear behavior, which makes their study challenging. No unified framework exists to control these robots, especially when considering their dynamics. This work proposes a methodology to study this type of robots around a stable equilibrium point. It can make the robot converge faster and with reduced oscillations to a desired equilibrium state. Using computational mechanics, a large-scale dynamic model of the robot is obtained and model reduction algorithms enable the design of low order controller and observer. A real robot is used to demonstrate the interest of the results.
\end{abstract}

Index Terms-Modeling, Control, and Learning for Soft Robots, Robust Control of Robotic Systems, Model Order Reduction

\section{INTRODUCTION}

O $\mathrm{NE}$ of the driving forces behind soft robotics is the requirement for machines that can work closely with humans rather than in competition with them. Where there is no need for a high level of adaptability in the environment, there is no need to think about soft robots; traditional rigid robots will most likely outperform soft robots if the desired task is well defined, in a well known and structured environment. The ever-increasing mastery of rigid robots and the desire to design more realistic robots, to interact with humans and/or in confined space in contacts with the environment, pushed the roboticist community to invent new paradigms, among which is soft robotics [1], [2], [3].

By definition, soft robots are compliant to the environment, and regarding the variety of materials used for their design and manufacture, which are also lighter than rigid robots. Another advantage is a larger power to weight ratio, which make them easy to deploy. Potential applications are too numerous to be all cited, but one of the most important is health-care industry, in particular surgery where a soft robot is used as a medical device for heart implant [4], cardiovascular therapy [5] and

Manuscript received: June, 22, 2018; Revised September, 13, 2018; Accepted October, 7, 2018.

This paper was recommended for publication by Editor Yu Sun upon evaluation of the Associate Editor and Reviewers' comments. This project has been funded by Universities of Lille and Valenciennes, by ANR (Project ANR17-ERC2-0029) and by the European Union through the European Regional Development Fund (ERDF).

${ }^{1}$ M. Thieffry and T.M. Guerra are with the university of Valenciennes, CNRS, UMR 8201 - LAMIH F-59313 Valenciennes, France maxime.thieffrydinria.fr

${ }^{2}$ M. Thieffry, A. Kruszewski and C. Duriez are in Defrost team, Inria, university of Lille, Centrale Lille, CRIStAL - Centre de Recherche en Informatique Signal et Automatique de Lille - UMR 9189, France

Digital Object Identifier (DOI): see top of this page. prosthesis and exosuits: removing rigid links in wearable robot makes it more comfortable to wear, and more efficient [6], [7].

Biology provides a major inspiration in the design of soft robots. The elephant's trunk, octopus arm or snake body lead to the design of the first soft robots [8], [9]. The octopus' arm's morphology allows it to benefit from its interaction with the environment to achieve a specific task. These biological examples have no backbone and achieve therefore better performance in unstructured and cluttered environments, which is one of the motivation to design soft robots. This bioinspiration can also come from the human, and helps to improve industrial gripper, or, coming back to health related applications, could improve the hand-prosthesis design [10].

This new field of research has opened research areas, among which is the need of accurate modeling methods, design tools and control methodologies [11], [12]. The present work aims at providing new results in this last category: it proposes a methodology to control the dynamics of soft robots around a stable equilibrium point based on its finite element model.

\section{PREVIOUS WORK AND STATE OF THE ART}

\section{A. Modeling}

Soft robotics is often referred to as a subclass of continuum robots, and results are available in this field to design, model and control continuum robots as in [13], and [14] presents a detailed state-of-the-art of kinematic modeling of continuum manipulator for which a visual summary is shown in figure 1 .

Soft robotics can take benefits of some methods initially developed for continuum robots, such as the Cosserat-Rod theory, see [15] for details. This theory has the advantage of being real-time computable and geometrically exact. Another modeling method is the Piecewise-Constant Curvature, see [16] for details, but it requires the studied robots to have a specific shape in order to validate the constant curvature assumption.

For simple geometry, such as beam, the modeling problem can be solved, as in [17]. However, the present work aims at being generic and should therefore not make any assumption regarding the geometry of the robot in order to be used on various types of robots.

Different modeling solutions are combined in [18], where authors use continuum robot modeling, finite element method (FEM) and machine learning to develop a generic modeling method for soft robots. Finite element method is also used in [19] and [20]. These papers propose a real-time implementation of the FEM that has the advantage to start from the constitutive law of the material which is measurable experimentally. 


\section{B. Control}

The aforementioned FEM methodology has shown some significant results for kinematics control: [14], [21]. However, the extension of this method to dynamical study is not trivial and brings additional challenges, such as dimensionality.

A recent survey recall the current state of research in control of soft robots manipulators [22]. With a precise comparison between model-based and model-free controllers and kinematics and dynamic controllers, this work states that considering the complete dynamic of the robots is probably the most challenging field of soft robot control. It recall that modelbased dynamic controllers are still in their "nascent stage and consequently, there are a multitude of gaps that should be addressed in design, modeling, and control". This is the aim of the present work.

Recently, different strategies to provide generic approaches for soft robot control have been proposed. In [23], authors propose a solution for the inverse kinematics problem and a model free control method is presented in [24]. This last method is based on machine learning and seems not to require any assumptions about the model, but is for now restricted to open-loop. Learning algorithms have also been used in [25] where a generic control framework is presented. The control strategy is based on a training step to learn the model without prior consideration regarding the robot's structure. In the present work, we aim at presenting a model-based dynamic control approach using finite element method modeling, such as in [26].

Soft robots have a theoretical infinite number of degrees of freedom. The spatial discretization in finite element limits it to a considerable large number and brings us to large-scale control theory, which is also an active field of research.

To design a controller usable in practice with a reasonable number of sensors, the use of model reduction is explored. Again, lots of results exists in this field, as recalled here [27], bringing the soft robotics community to use it for soft robot modeling [28], and control of continuum manipulators [29].

In [30], authors identify difficulties about soft robot control because identifying good performance is not straightforward when studying soft robots. This work highlights the need of a better control design but shows that those controllers affect the natural behavior of the soft robot, making them stiffer. A mix between feedback, feedforward and iterative learning is proposed to solve this issue.

\section{PRESENT CONTRIBUTION}

The present work aims at providing a methodology to control soft robots in order to make them converge faster and without oscillations to a desired final position. It is an extension of the work presented in [26], that presented a methodology to control the convergence rate of soft robots using state feedback controller. The present work extends the previous results from state feedback (all states were supposed available) to output feedback (real-time considerations) via a state observer design. The full methodology is then tested experimentally. It covers modeling, control and state observation of soft robots in both simulation and real experiments on an

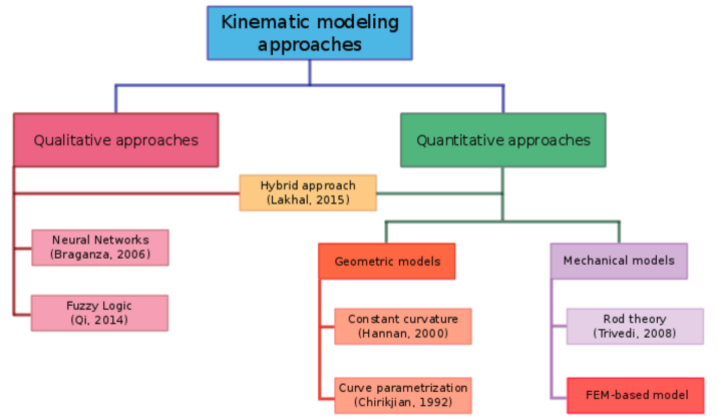

Fig. 1. Summary of available modeling methods for continuum robots, from [14]

academic case. It focuses on model-based control, to maximize the benefits from the FEM method described in [19].

To avoid the expansion of dimensionality and ensure the solvability of the problem two steps are performed. The first one is to get a linear model from the FEM nonlinear model via linearization. The second step uses model-reduction providing a reduced linear model compatible with the actual solvers, in order to derive both a controller and an observer (for the unmeasured states). Of course, both steps introduce an error that has to be taken into account for the control design.

The present work contributions are:

- an experimental validation of the control methodology presented in [26],

- a robust reduced order Luenberger observer.

\section{FEM-BASED MODELING}

\section{A. FEM model}

Modeling soft robots relies on both continuum mechanics theory and numerical approaches to solve the underlying equations. First, it requires a mesh of the studied robot, which can be obtained using any mechanical design software. Let us define position and velocity vectors, respectively $q(t) \in \mathbb{R}^{n}$ and $v(t) \in \mathbb{R}^{n}$ whose dimension $n$ is proportional to the size of the FEM mesh used to model the robot. As there is no ambiguity, the time-dependency of those vectors will be omitted in the latter and $v(t)=v, q(t)=q$. The more nodes the mesh has, the more the model tends to be accurate and, for soft robots application, the size $\mathcal{N}$ of the FEM mesh goes from hundreds to thousands of variables. The dimension $n$ of the vectors $q$ and $v$ is made of $3 \times \mathcal{N}$ variables, as the position and velocity vectors are given in the 3 dimension of space.

The non-linear equation of motion of the robots is given by Newton's law:

$$
\mathcal{M}(q) \dot{v}=\mathcal{P}(q)-\mathcal{F}(q, v)+\mathcal{H}^{T}(q) z(t)
$$

where $\mathcal{M}(q)$ is the mass matrix, $\mathcal{H}^{T}(q) z$ is the actuators contribution : $\mathcal{H}^{T}(q)$ contains the direction of the actuators forces and $z$ their amplitude. The matrix $\mathcal{F}(q, v)$ represents the internal forces and $\mathcal{P}(q)$ gathers all the known external forces. As we consider only the gravity field, $\mathcal{P}(q)$ is constant and $\mathcal{P}(q)=\mathcal{P}$.

Let $q_{0} \in \mathbb{R}^{n}$ be a stable equilibrium that could be obtained using an inverse problem. This configuration $q_{0}$ is the desired 
state of the robot and is induced by $\mathcal{P}$ and $z(t)=z_{0}$, i.e. $q_{0}$ is solution to

$$
0=\mathcal{P}-\mathcal{F}\left(q_{0}, 0\right)+\mathcal{H}^{T}\left(q_{0}\right) z_{0}
$$

Equation (1) can also be written as:

$$
\begin{aligned}
\mathcal{M}(q) \dot{v} & =\mathcal{P}-\mathcal{F}(q, v)+\mathcal{H}^{T}(q) u(t)-\mathcal{P}+\mathcal{F}\left(q_{0}, 0\right)-\mathcal{H}^{T}\left(q_{0}\right) z_{0} \\
\Leftrightarrow & \\
\mathcal{M}(q) \dot{v} & =\mathcal{F}\left(q_{0}, 0\right)-\mathcal{F}(q, v)+\mathcal{H}^{T}(q) u(t)-\mathcal{H}^{T}\left(q_{0}\right) z_{0}
\end{aligned}
$$

We can approximate the internal forces $\mathcal{F}$ with a first order Taylor expansion around this equilibrium point:

$$
\mathcal{F}(q, v) \approx \mathcal{F}\left(q_{0}, 0\right)+\left.\frac{\partial \mathcal{F}(q, 0)}{\partial q}\right|_{q=q_{0}}\left(q-q_{0}\right)+\left.\frac{\partial \mathcal{F}\left(q_{0}, v\right)}{\partial v}\right|_{v=0}
$$

where $\frac{\partial \mathcal{F}(q, v)}{\partial q}=\mathcal{K}(q, v)$ is the compliance matrix, and $\frac{\partial \mathcal{F}(q, v)}{\partial v}=\mathcal{D}(q, v)$ is the damping matrix. By definition, mass, compliance and damping matrices are positive definite. With these notations, the equation of motion around an equilibrium point $q_{0}$ is given by the following relation:

$$
\mathcal{M}(q) \dot{v} \approx-\mathcal{K}\left(q_{0}, 0\right) d-\mathcal{D}\left(q_{0}, 0\right) v+\mathcal{H}^{T}(q) u(t)-\mathcal{H}^{T}\left(q_{0}\right) z_{0}
$$

with $d$ the displacement vector defined by:

$$
d=q-q_{0}
$$

\section{B. Linear state-space Equation}

Around an equilibrium point $q_{0}$, let us consider $\mathcal{M}=$ $\mathcal{M}\left(q_{0}, 0\right), \mathcal{D}=\mathcal{D}\left(q_{0}, 0\right), \mathcal{K}=\mathcal{K}\left(q_{0}, 0\right)$ and $\mathcal{H}\left(q_{0}\right)=\mathcal{H}(q)=$ $\mathcal{H}$. Defining the new input vector $u$ as $u=z-z_{0}$, system (5) can be approximated by the following linear representation:

$$
\left\{\begin{array}{l}
\dot{x}=\underbrace{\left(\begin{array}{cc}
-\mathcal{M}^{-1} \mathcal{D} & -\mathcal{M}^{-1} \mathcal{K} \\
I & 0
\end{array}\right)}_{A} x+\underbrace{\left(\begin{array}{c}
\mathcal{M}^{-1} \mathcal{H}^{T} \\
0
\end{array}\right)}_{B} u \\
y=C x
\end{array}\right.
$$

where $x=\left(\begin{array}{l}v \\ d\end{array}\right), x \in \mathbb{R}^{2 n}$ and where system matrices are large-scale sparse matrices, i.e. $A \in \mathbb{R}^{2 n \times 2 n}, B \in$ $\mathbb{R}^{2 n \times m}, C \in \mathbb{R}^{p \times 2 n}$, where $m$ is the number of actuators and $p$ the number of outputs.

\section{Experiments on a real robot}

1) Experimental setup: The theoretical results developed in this paper are illustrated with an experimental setup shown in figure 2 and 3. This robot is a 90 centimeters long soft beam that weighs 13 grams. It is actuated with 2 cables placed at the middle of the beam, at 45 centimeter of the wall. The validation results are given for the following experiment: the robot is released from an initial position, left of figure 2, and converges to a stable position given by $P$ and $u_{0}$, right of figure 2. The output of this robot is the displacement of the tip (left extremity) of the beam in the three directions of space, $x y$ and $\mathrm{z}$ as described on the figure 3 . In simulation, a mesh made of 2531 tetrahedrons and 907 nodes is used, corresponding to position and velocity vectors of dimension $n=2721$ and a state $x \in \mathbb{R}^{5442}$.
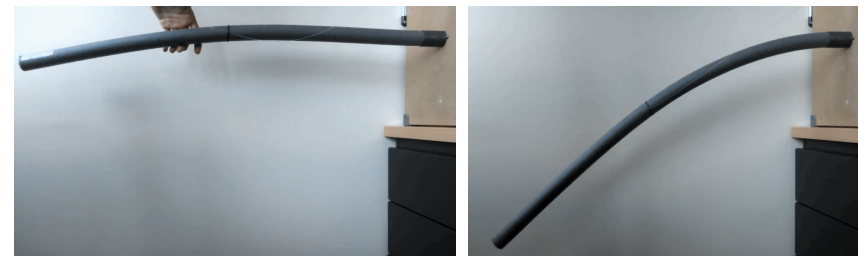

Fig. 2. Experimental conditions. Left: start position; Right: end position

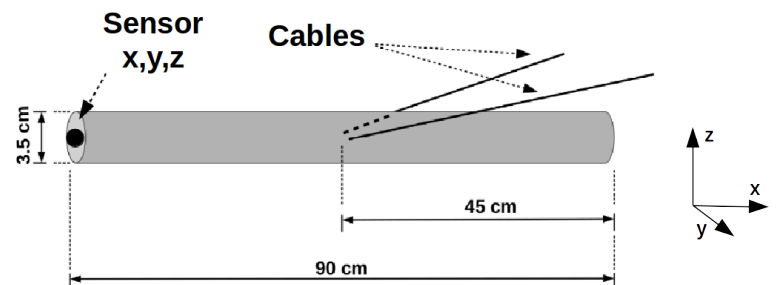

Fig. 3. Schematic view of the robot

The displacement of the tip of the beam in open-loop is shown in figure 4.

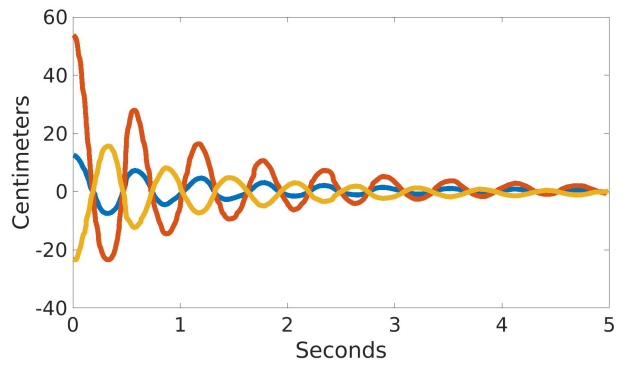

Fig. 4. Measurements of open-loop position error of the tip of the robot. Red : error along $\mathrm{z}$ axis; Yellow : $\mathrm{x}$ axis; Blue : $\mathrm{y}$ axis.

2) Nonlinear model validation: Using the SOFA framework, see [19] for details, the nonlinear system is simulated and its output is compared to the real measurements in figure 5. It presents the worst case error signal evolution compared to the real measurement, with a 3.5 centimeters absolute maximum error.

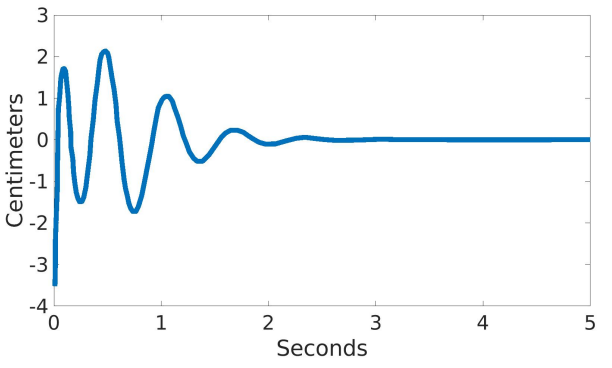

Fig. 5. Difference between outputs of nonlinear model and real robot.

3) Validation of the linearization step: Figure 6 presents the worst case error signal between linear and nonlinear evolution with a 1.1 centimeters absolute maximum error. In addition, the linear model is validated as it captures faithfully the oscillations. 


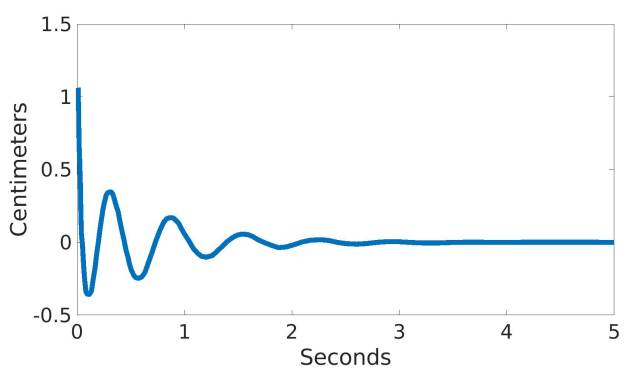

Fig. 6. Difference between outputs of nonlinear and linear models.

\section{MODEL ORDER REDUCTION}

To design a state feedback control law, the large-scale FEM model is not adapted. Control design tools are indeed not suitable for systems with thousands of decision variables. That is why model reduction is proposed to obtain a system of reduced dimension used to compute both controller and observer.

Even if different results exist in the literature for linear model reduction, like balanced truncation or iterative tangential interpolation [31], the only method suitable for non-linear system is the Proper Orthogonal Decomposition (POD) [27]. To ease the extension of this work to nonlinear model, this method is used to perform model reduction.

\section{A. State projection}

Projection-based model order reduction consists of finding two projectors $V=\left(\begin{array}{ll}V_{r} & V_{\bar{r}}\end{array}\right) \in \mathbb{R}^{2 n \times 2 n}$ and $W=$ $\left(\begin{array}{ll}W_{r} & W_{\bar{r}}\end{array}\right) \in \mathbb{R}^{2 n \times 2 n}$ to compute a reduced order state $x_{r} \in \mathbb{R}^{r}$ and a neglected state $x_{\bar{r}} \in \mathbb{R}^{2 n-r}$, defined as:

$$
x=V_{r} x_{r}+V_{\bar{r}} x_{\bar{r}}
$$

Projectors are orthogonals, it holds $W_{r}^{T} V_{r}=I$ and $W_{r}^{T} V_{\bar{r}}=0$. Thus, the system's dynamics writes:

$$
\left\{\begin{array}{l}
\dot{x}_{r}=\underbrace{W_{r}^{T} A V_{r}}_{A_{r}} x_{r}+\underbrace{W_{r}^{T} B}_{B_{r}} u+W_{r}^{T} A V_{\bar{r}} x_{\bar{r}} \\
\dot{x}_{\bar{r}}=W_{\bar{r}}^{T} A V_{\bar{r}} x_{\bar{r}}+W_{\bar{r}}^{T} B u+W_{\bar{r}}^{T} A V_{r} x_{r} \\
y_{r}=\underbrace{C V}_{C_{r}} x+C V_{\bar{r}} x_{\bar{r}}
\end{array}\right.
$$

It provides us with a low dimensional state $x_{r}$ and a reduced order system which is used to design a controller and an observer. In both case, the aim is to use only $x_{r}$ to control the whole state dynamics described in (7).

\section{B. Application to soft robotics}

POD reduction needs a first experiments where snapshots of the robots' state are saved, then a singular value decomposition of these snapshots is performed. The state vector is truncated depending on how fast the singular values decay. The presented reduction method is used on the soft robot presented in figure 2. Figure 7 shows the decay of its singular values, showing a fast decay for the first 10 values, and those 10 first values represent more than $99 \%$ of the singular values of the model. The results of the reduction algorithm are shown in figure 8, that shows a comparison between outputs of the full and reduced systems with 10 states. The reduced system clearly represents faithfully the large-order one around the studied equilibrium point.

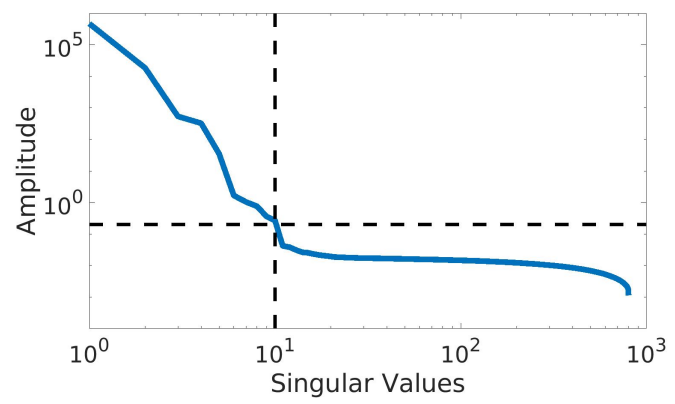

Fig. 7. Evolution of the singular values of the model of the soft robot.

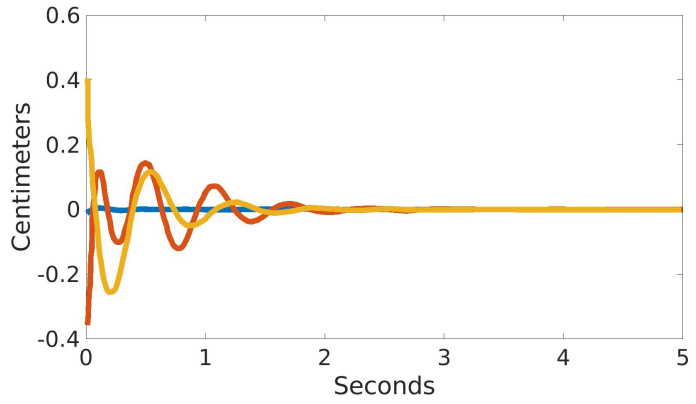

Fig. 8. Error between outputs of large and reduced models $\frac{y_{r}-y}{\|y\|}$. Red : error along $\mathrm{z}$ axis; Yellow : $\mathrm{x}$ axis; Blue : y axis.

\section{CONTROL DESIGN}

The aim of this work is to present a methodology to design feedback controller for soft robots, based on the dynamical model presented in the previous section. Robots' dynamics is controlled based on the reduced order model introduced previously. A control method for soft robots based on a reduced model and taking into account reduction error has been presented in [26]. The idea behind this control design is to control the dynamics of the reduced system using a feedback control law and ensure the large-scale closed loop stability using Lyapunov theory.

Let $L \in \mathbb{R}^{m \times r}$, where $m$ is the number of inputs of the system, be the feedback matrix of the control law:

$$
u=-L x_{r}
$$

leading to the following closed-loop reduced order model:

$$
\left\{\begin{array}{l}
\dot{x}_{r}=\left(A_{r}-B_{r} L\right) x_{r} \\
y_{r}=C_{r} x_{r}
\end{array}\right.
$$

and to corresponding the large-scale closed-loop system:

$$
\left\{\begin{array}{l}
\dot{x}=\left(A-B L W^{T}\right) x \\
y=C x
\end{array}\right.
$$


Let us recall here theorem 2 of [26]. It is based on the existence of an energy-based Lyapunov function in open-loop. The closed-loop poles are placed at desired location thanks to the state feedback and the stability is ensured thanks to the large-scale LMI.

Theorem 2: System (7) with feedback (10) is stable with a decay rate $\lambda$ if:

There exists $\epsilon$ such as:

$$
\left(\begin{array}{cc}
(1+\epsilon) \mathcal{M} & \epsilon \mathcal{M} \\
\epsilon \mathcal{M} & (1+\epsilon) \mathcal{K}+\epsilon \mathcal{D}
\end{array}\right)>0
$$

and

$$
(\mathbf{S}+\mathbf{T})<-\lambda\left(\begin{array}{cc}
(1+\epsilon) \mathcal{M} & \epsilon \mathcal{M} \\
\epsilon \mathcal{M} & (1+\epsilon) \mathcal{K}+\epsilon \mathcal{D}
\end{array}\right)
$$

with

$$
\mathbf{S}=V^{T}\left(\begin{array}{cc}
-(1+\epsilon) \mathcal{D}+\epsilon \mathcal{M} & 0 \\
0 & -\epsilon \mathcal{K}
\end{array}\right) V+(*)
$$

which also writes

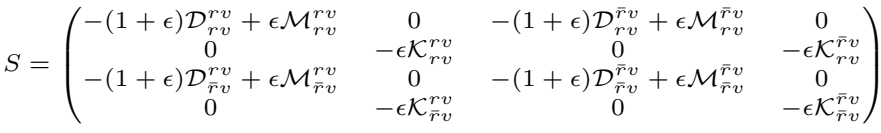

where $\mathcal{D}_{r v}^{r v}=V_{r v}^{T} \mathcal{D} V_{r v}, \mathcal{D}_{r v}^{\bar{r} v}=V_{r v}^{T} \mathcal{D} V_{\bar{r} v}$ and

$$
\begin{aligned}
\mathbf{T} & =V^{T}\left(\begin{array}{c}
(1+\epsilon) \mathcal{H}^{T} \\
\epsilon \mathcal{H}^{T}
\end{array}\right) L V+(*) \\
& =\left(\begin{array}{cccc}
(1+\epsilon) V_{r v}^{T} \mathcal{H}^{T} L_{r v} & (1+\epsilon) V_{r v}^{T} \mathcal{H}^{T} L_{r d} & 0 & 0 \\
\epsilon V_{r d}^{T} \mathcal{H}^{T} L_{r v} & \epsilon V_{r d}^{T} \mathcal{H}^{T} L_{r d} & 0 & 0 \\
(1+\epsilon) V_{\bar{r} v}^{T} \mathcal{H}^{T} L_{r v} & (1+\epsilon) V_{\bar{r} v}^{T} \mathcal{H}^{T} L_{r d} & 0 & 0 \\
\epsilon V_{\bar{r} d}^{T} \mathcal{H}^{T} L_{r v} & \epsilon V_{\bar{r} d}^{T} \mathcal{H}^{T} L_{r d} & 0 & 0
\end{array}\right)+(*)
\end{aligned}
$$

This LMI problem implies few decision variables, as the feedback matrix $L$ is of reduced dimension, and a large number of constraints are solved to ensure large-scale stability and performances. In the case considered in this work, the state vector $x$ is made of 5442 variables, the matrices $\mathbf{S}$ and $\mathbf{T}$ are also of dimension $5442 \times 5442$ and the low order feedback matrix $L$ is of dimension $2 \times 10$.

This control strategy is tested in simulation on the linear large-scale system. The goal of this simulation experiment is to drive the robot to the final position with reduced vibrations, compared to figure 4 . Results are shown on the figure 9 and show a clear diminution of the oscillations.

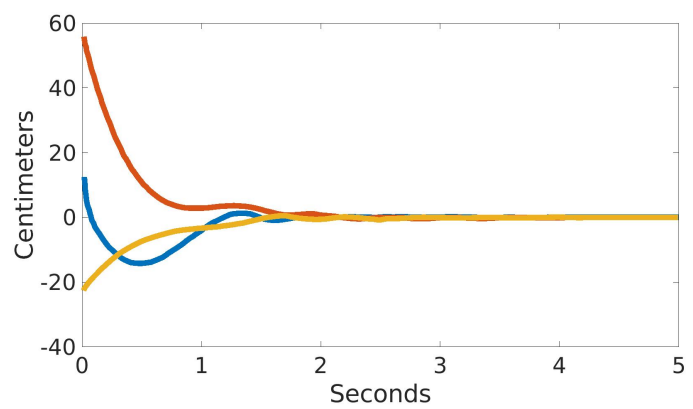

Fig. 9. Position error of the large-scale linear model in closed-loop. Red : error along $\mathrm{z}$ axis; Yellow : $\mathrm{x}$ axis; Blue : $\mathrm{y}$ axis.

\section{OBSERVER}

To use the previous control design in practice, where neither $x$ nor $x_{r}$ are available from sensor, an observer is designed to reconstruct $x_{r}$ from $y$ (in our case, from the position of the tip in three dimensions of space). As for the control part, where the reduction error is taken into account in the computation, the goal is to design an observer that is robust according to the reduction error. To do so, an unknown input observer, in the form of a PI-observer as in [32], reconstructs $x_{r}$ and considers the reduction error as a disturbance, i.e. the unknown input.

\section{A. Observer design}

From (9), the dynamics of the reduced order state writes:

$$
\left\{\begin{array}{l}
\dot{x}_{r}=A_{r} x_{r}+B_{r} u+W_{r}^{T} A V_{\bar{r}} x_{\bar{r}} \\
y_{r}=C_{r} x_{r}
\end{array}\right.
$$

A Luenberger-observer for this system would be:

$$
\begin{aligned}
& \dot{\hat{x}}_{r}=A_{r} \hat{x}_{r}+B_{r} u+W_{r}^{T} A V_{\bar{r}} x_{\bar{r}}+F_{o b s}\left(y_{r}-\hat{y}_{r}\right) \\
& \hat{y}_{r}=C_{r} \hat{x}_{r}
\end{aligned}
$$

However, the vector $x_{\bar{r}} \in \mathbb{R}^{2 n-r}$ is of large dimension and cannot be used to compute $F_{\text {obs }}$. Let us denote $\Phi=$ $W_{r}^{T} A V_{\bar{r}} x_{\bar{r}}, \Phi \in \mathbb{R}^{r}$, equation (17) writes:

$$
\left\{\begin{array}{l}
\dot{x}_{r}=A_{r} x_{r}+B_{r} u+\Phi \\
y_{r}=C_{r} x_{r}
\end{array}\right.
$$

We propose to define an unknown input $\omega$ to model the behavior of $\Phi$. Let us assume that:

$$
\Phi=Z \omega+\Psi
$$

with $Z \in \mathbb{R}^{r \times n_{\omega}}, \omega \in \mathbb{R}^{n_{\omega}}$ and $\Psi \in \mathbb{R}^{r}$ and assuming that the $p^{t h}$ derivative of $\omega$ is zero, i.e. the dynamics of $\omega$ can be captured via a cascade of integrators such as:

$$
\underbrace{\left(\begin{array}{c}
\dot{\omega} \\
\ddot{\omega} \\
\vdots \\
\omega^{(p)}
\end{array}\right)}_{\check{\Gamma}}=\underbrace{\left(\begin{array}{cccc}
0 & 1 & \ldots & 0 \\
\vdots & \ddots & \ddots & 0 \\
0 & \ldots & 0 & 1 \\
0 & \ldots & \ldots & 0
\end{array}\right)}_{J} \underbrace{\left(\begin{array}{c}
\omega \\
\dot{\omega} \\
\vdots \\
\omega^{(p-1)}
\end{array}\right)}_{\Gamma}
$$

Under these assumptions, (19) is observable iff:

$$
\left\{\begin{array}{c}
\left(A_{r}, C_{r}\right) \text { is observable, } \\
\operatorname{rank}(Z) \leq n_{y}
\end{array}\right.
$$

where $n_{y}$ is the number of outputs. The problem is that $\Phi$ is of larger dimension than $\omega$, otherwise the state would be completely measured. A way to tackle this problem is to determine the matrix $Z$ that minimizes $\Phi-Z \omega$ and that can be obtained thanks to simulation experiments, where the vector $\Phi$ is computable and snapshots of this vector are saved:

$$
\Omega=\left(\begin{array}{lllll}
\Phi\left(t_{1}\right) & \Phi\left(t_{2}\right) & \Phi\left(t_{3}\right) & \ldots & \Phi\left(t_{f}\right)
\end{array}\right)
$$

It is possible to see how $x_{\bar{r}}$ affects $x_{r}$ by performing a singular value decomposition of $\Omega$ and retrieve the unknown input matrix $Z$ : 


$$
\Omega=Z\left(\operatorname{diag}\left(\sigma_{i}\right) \quad 0_{2 n \times(t f-2 n)}\right)\left(\begin{array}{c}
\omega_{1} \\
\omega_{2} \\
\vdots \\
\omega_{t f}
\end{array}\right)
$$

Where $\sigma_{i}$ are the singular values of $\Omega$ and $0_{r, c}$ is a zero matrix of size $r \times c$. Thus, for each $t_{i}$ the reduction error is:

$$
\Omega_{i}=\Phi\left(t_{i}\right)=Z\left(\operatorname{diag}\left(\sigma_{i}\right) \quad 0_{2 n \times(t f-2 n)}\right) \omega_{i}
$$

which also writes:

$$
\begin{aligned}
& \Phi\left(t_{i}\right)=\sum_{j=1}^{2 n} Z_{j} \sigma_{j} \omega_{i, j} \\
& \Phi\left(t_{i}\right)=\sum_{j=1}^{r} Z_{j} \sigma_{j} \omega_{i, j}+\sum_{j=r+1}^{2 n} Z_{j} \sigma_{j} \omega_{i, j}
\end{aligned}
$$

From SVD algorithm, it holds:

$$
\left\|\sum_{j=r+1}^{2 n} Z_{j} \sigma_{j} \omega_{i, j}\right\| \leq \sum_{j=r+1}^{2 n} \sigma_{j}
$$

The vector $\omega$ gathers the reduction error and is the unknown input of the observer.

$$
\begin{aligned}
& \dot{\hat{x}}_{r}=A_{r} \hat{x}_{r}+B_{r} u+F_{o b s}\left(y-\hat{y}_{r}\right)+Z \hat{\omega} \\
& \hat{y}_{r}=C_{r} \hat{x}_{r}
\end{aligned}
$$

Considering the extended state $x_{e}=\left(\begin{array}{ll}x_{r} & \Gamma\end{array}\right)^{T}$, whose dynamics (with $\Gamma$ and $J$ defined in (21)) writes:

$$
\dot{x}_{e}=\underbrace{\left(\begin{array}{cccc}
A_{r} & \left(\begin{array}{llll}
Z & 0 & \ldots & 0
\end{array}\right) \\
0 & & J
\end{array}\right)}_{A_{e}} x_{e}+\underbrace{\left(\begin{array}{c}
B r \\
0
\end{array}\right)}_{B_{e}} u+\left(\begin{array}{c}
\Psi \\
0
\end{array}\right)
$$

and the observed extended state

$$
\hat{x}_{e}=\left(\begin{array}{c}
\hat{x}_{r} \\
\hat{\Gamma}
\end{array}\right)
$$

the observation error $e=x_{e}-\hat{x}_{e}$ dynamics is the following:

$$
\dot{e}=\left(\begin{array}{ccccc}
A_{r}-F_{o b s_{1}} C_{r} & \left(\begin{array}{llll}
Z & 0 & \ldots & 0
\end{array}\right) \\
-F_{o b s_{2}} C_{r} & & & J &
\end{array}\right) e\left(\begin{array}{c}
\Psi \\
0
\end{array}\right)
$$

with $\|\Psi\| \leq \sum_{j=r+1}^{2 n} \sigma_{j}$.

In order to minimize the effect of the residue $\Psi$, the observer gain $F_{o b s}=\left(\begin{array}{ll}F_{o b s_{1}} & F_{o b s_{2}}\end{array}\right)^{T}$ is computed using a $\mathcal{H}_{\infty}$ criteria:

$$
F_{o b s}=\underset{F_{o b s}}{\arg \min } \max _{\|\Psi\|<1} \frac{\|e\|_{2}}{\|\Psi\|_{2}}
$$

\section{B. Simulation results}

During this simulation experiment, the observed state $\hat{x}_{r}$ is compared to the one of large-scale linear system (7), i.e. $W_{r}^{T} x$, the corresponding results are shown in figure 10 .

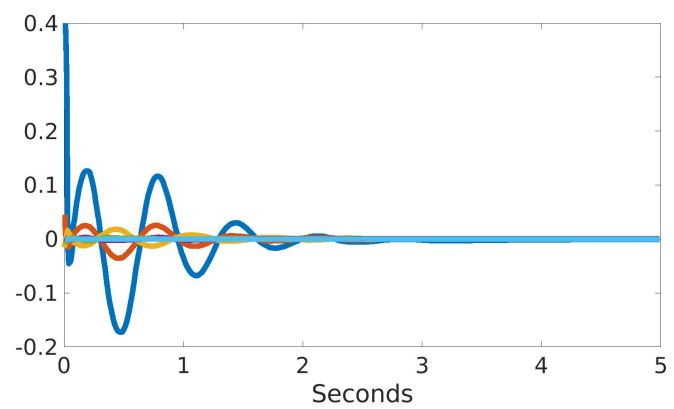

Fig. 10. Error between projected state of large linear model and observed state: $\frac{W_{r}^{T} x-\hat{x}_{r}}{\left\|W^{T} x\right\|}$.

\section{Experiments on real device}

Once both controller and observer have been tested in simulation, the entire control strategy, i.e. low dimensional state feedback using observer, is applied to the real robot and results are shown in figure 11. Compared to figure 4, it shows a clear diminution of the oscillations before converging to the reference. However, the oscillations are not exactly damped as it was noticed during simulation. This behavior is mainly due to the state estimation error dynamics.

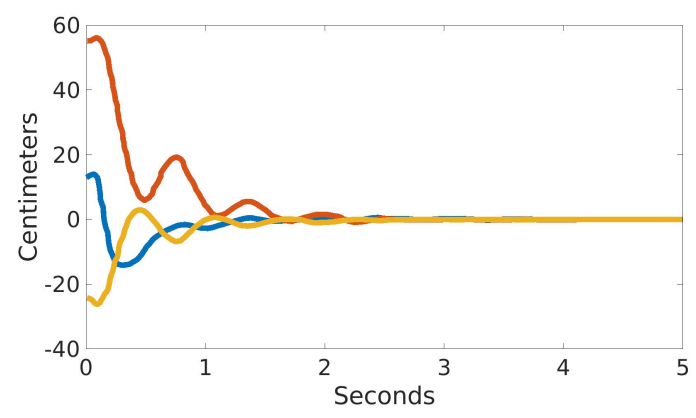

Fig. 11. Measurements of position error of the tip of the beam in closed-loop. Red : error along $\mathrm{z}$ axis; Yellow : $\mathrm{x}$ axis; Blue : $\mathrm{y}$ axis.

Several experiments were done and the results of four of them are given in table I. The Integral Time Absolute Error (ITAE), defined as ITAE $=\int_{0}^{T} t|e(t)| d t$, and the $3 \%$-settling-time are provided in the table to compare open and closed-loop results. These experiments were made with the same setup as described in part IV-C1, with different initial positions around an equilibrium point. Experiments 1 and 2 correspond to the following experiment: the robot is released from an initial position of $(x, y, z)=(-22,12,53)$ as shown in figure 2 , and for experiments 3 and 4 the robot is released from an initial position $(x, y, z)=(-18 ;-2 ; 35)$.The results show an average diminution of $40 \%$ for the settling time and $30 \%$ for the ITAE. 


\begin{tabular}{|c|c|c|c|c|}
\hline & Exp1 & Exp2 & Exp3 & Exp4 \\
\hline Open & ITAE $=1795$ & ITAE $=1675$ & $\mathrm{ITAE}=1420$ & ITAE $=1350$ \\
\hline loop & $\mathrm{ST}=5.4 \mathrm{~s}$ & $\mathrm{ST}=4.9 \mathrm{~s}$ & $\mathrm{ST}=4.1 \mathrm{~s}$ & $\mathrm{ST}=4 \mathrm{~s}$ \\
\hline Closed & ITAE 1312 & ITAE $=1102$ & ITAE $=1110$ & ITAE $=1090$ \\
\hline loop & $\mathrm{ST}=3.3 \mathrm{~s}$ & $\begin{array}{c}\mathrm{ST}=2.9 \mathrm{~s} \\
\text { TABLE I }\end{array}$ & $\mathrm{ST}=2.8 \mathrm{~s}$ & $\mathrm{ST}=2.6 \mathrm{~s}$ \\
\hline
\end{tabular}

Another set of experiments is conducted on this robot. The goal is to make the tip of the beam follow a trajectory (see attached video). Results are shown in figure 12 that displays the comparison between the reference trajectory (in red) and the measurements of the position of the tip of the beam along the $\mathrm{z}$ axis. Even if oscillations are present along the reference trajectory, the proposed controller enables the control of the robot along desired paths.

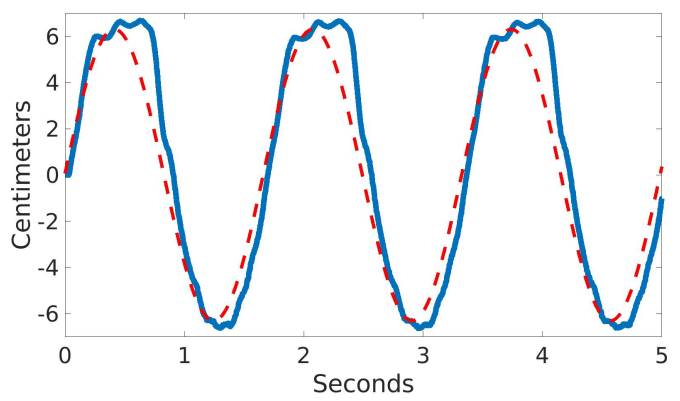

Fig. 12. Measurements of the position of the beam along the $\mathrm{z}$ axis. Red : reference, Blue $=$ real measurements.

\section{COMPLETE CLOSED-LOOP STABILITY ANALYSIS}

This section is dedicated to the study of the stability of the closed-loop using the observer, i.e. the stability of system (33):

$$
\dot{x}=A x-B L \hat{x}_{r}
$$

which can be written as:

$$
\left(\begin{array}{c}
\dot{x}_{r} \\
\dot{x}_{\bar{r}}
\end{array}\right)=\left(\begin{array}{ll}
W_{r}^{T} A V_{r}^{T} & W_{r}^{T} A V_{\bar{r}}^{T} \\
W_{\bar{r}}^{T} A V_{r}^{T} & W_{\bar{r}}^{T} A V_{\bar{r}}^{T}
\end{array}\right)\left(\begin{array}{c}
x_{r} \\
x_{\bar{r}}
\end{array}\right)+\left(\begin{array}{l}
W_{r}^{T} B \\
W_{\bar{r}}^{T} B
\end{array}\right) L \hat{x}_{r}
$$

and using the definition of $e=x_{e}-\hat{x}_{e}$, whose dynamics is described in equation (31), the previous equation can be written in the following form:

$$
\left(\begin{array}{c}
\dot{x}_{r} \\
\dot{x}_{\bar{r}} \\
\dot{x}_{r}-\dot{\hat{x}}_{r} \\
\dot{\Gamma}-\dot{\hat{\Gamma}}
\end{array}\right)=\mathcal{O}\left(\begin{array}{c}
x_{r} \\
x_{\bar{r}} \\
x_{r}-\hat{x}_{r} \\
\Gamma-\hat{\Gamma}
\end{array}\right)
$$

where the matrix $\mathcal{O}$ is defined as:

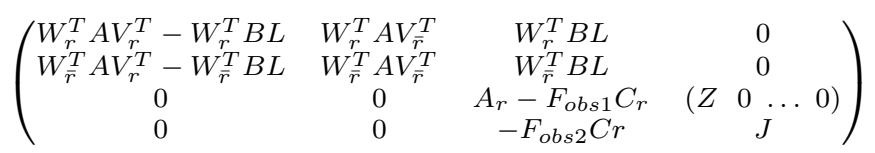

This formulation can be used as a tool to check the stability of the complete closed-loop system, under the assumption that the reduction error is bounded. Once both controller and observer have been computed using the methodology described in the previous sections, the sign of the eigenvalues of $\mathcal{O}$ determines the stability of the closed-loop.

In the case of the foam beam studied in this work, the 5455 eigenvalues of $\mathcal{O}$ are all negative, with a maximum real part of -7.7058 . The closed-loop system composed of the large-scale model of section IV, the observer designed in section VII and the controller of section VI is also stable.

\section{DISCUSSION}

\section{A. Use of linear model}

The model used to compute both controller and observer is a linear model. Therefore, all the results presented in this paper are valid in a neighborhood around the equilibrium point where the system has been linearized. We are currently investigating the extension of this work to Linear Parameter Varying modeling, to enable the control around multiple points of interest.

\section{B. Different actuation and sensing}

The presented methodology has been tested on a real device to show its effectiveness. The soft robot considered in this work is actuated with cables and only the position of the tip is measured. In a near future, the proposed method will be implemented on several kind of soft robots in order to test how the method is generic, especially thinking that no geometric assumptions are needed as only a finite element mesh is needed. The proposed method can be tested indifferently on a robot actuated with cables, pneumatic, hydraulics... Only the definition of the matrix B of the model would be changed.

\section{No proof for tracking}

As the initial FEM nonlinear model is linearized around an equilibrium point, the trajectories to follow must remain in a region around this point. Moreover, the trajectory tracking is only tested experimentally and no theoretical proof has been given. This will be the topic of future research.

\section{Simultaneous Observer and Controller Design}

In the last section of this work, a validation of both controller and observer is proposed to check the stability of the closed-loop. It provides a proof of stability for the large-scale closed-loop system but is for now only a postdesign step. Future work will focus on the simultaneous design of both controller and observer while ensuring stability and performances of the large-scale closed-loop.

\section{CONCLUSIONS}

We have presented a control method for cancelling the vibrations of soft robots around a stable equilibrium point. This strategy uses finite element method and model reduction to compute a control law and an observer. Using Lyapunov theory and large-scale linear matrix inequalities, a reduced order feedback set the performances and ensure stability of the large-scale model in closed-loop. 
This work can be seen as a first step to design closedloop controller for soft robots using FEM and model order reduction. In addition to the tracks developed in the discussion section, future work could for instance include the ability to handle contacts with environment.

\section{REFERENCES}

[1] S. Kim, C. Laschi, and B. Trimmer, "Soft robotics: a bioinspired evolution in robotics," Trends in biotechnology, vol. 31 , no. 5, pp. $287-$ 294, 2013.

[2] C. Majidi, "Soft robotics: a perspective current trends and prospects for the future," Soft Robotics, vol. 1, no. 1, pp. 5-11, 2014.

[3] C. Laschi and M. Cianchetti, "Soft robotics: new perspectives for robot bodyware and control," Frontiers in bioengineering and biotechnology, vol. 2, p. 3, 2014.

[4] C. J. Payne, I. Wamala, C. Abah, T. Thalhofer, M. Saeed, D. BautistaSalinas, M. A. Horvath, N. V. Vasilyev, E. T. Roche, F. A. Pigula et al., "An implantable extracardiac soft robotic device for the failing heart: Mechanical coupling and synchronization," Soft robotics, vol. 4, no. 3, pp. 241-250, 2017.

[5] I. Wamala, E. T. Roche, and F. A. Pigula, "The use of soft robotics in cardiovascular therapy," Expert Review of Cardiovascular Therapy, vol. 15, no. 10, pp. 767-774, 2017, pMID: 28817983. [Online]. Available: https://doi.org/10.1080/14779072.2017.1366313

[6] Y. Ding, I. Galiana, A. T. Asbeck, S. M. M. De Rossi, J. Bae, T. R. T. Santos, V. L. de Araújo, S. Lee, K. G. Holt, and C. Walsh, "Biomechanical and physiological evaluation of multi-joint assistance with soft exosuits," IEEE Transactions on Neural Systems and Rehabilitation Engineering, vol. 25, no. 2, pp. 119-130, 2017.

[7] C. T. O’Neill, N. S. Phipps, L. Cappello, S. Paganoni, and C. J. Walsh, "A soft wearable robot for the shoulder: Design, characterization, and preliminary testing," in Rehabilitation Robotics (ICORR), 2017 International Conference on. IEEE, 2017, pp. 1672-1678.

[8] S. Neppalli, B. Jones, W. McMahan, V. Chitrakaran, I. Walker, M. Pritts, M. Csencsits, C. Rahn, and M. Grissom, "Octarm-a soft robotic manipulator," in Intelligent Robots and Systems, 2007. IROS 2007. IEEE/RSJ International Conference on. IEEE, 2007, pp. 2569-2569.

[9] C. Laschi, M. Cianchetti, B. Mazzolai, L. Margheri, M. Follador, and P. Dario, "Soft robot arm inspired by the octopus," Advanced Robotics, vol. 26, no. 7, pp. 709-727, 2012.

[10] E. Mattar, "A survey of bio-inspired robotics hands implementation: New directions in dexterous manipulation," Robotics and Autonomous Systems, vol. 61, no. 5, pp. 517-544, 2013.

[11] D. Rus and M. T. Tolley, "Design, fabrication and control of soft robots," Nature, vol. 521, no. 7553, p. 467, 2015.

[12] H. Lipson, "Challenges and opportunities for design, simulation, and fabrication of soft robots," Soft Robotics, vol. 1, no. 1, pp. 21-27, 2014.

[13] I. D. Walker, "Continuous backbone continuum robot manipulators," Isrn robotics, vol. 2013, 2013.

[14] T. Morales Bieze, "Contribution to the kinematic modeling and control of soft manipulators using computational mechanics," Ph.D. dissertation, Lille 1, 2017.

[15] F. Renda, F. Boyer, J. Dias, and L. Seneviratne, "Discrete cosserat approach for multi-section soft robots dynamics," arXiv preprint arXiv:1702.03660, 2017.

[16] R. J. Webster III and B. A. Jones, "Design and kinematic modeling of constant curvature continuum robots: A review," The International Journal of Robotics Research, vol. 29, no. 13, pp. 1661-1683, 2010.

[17] B. Gamus, L. Salem, E. Ben-Haim, A. D. Gat, and Y. Or, "Interaction between inertia, viscosity, and elasticity in soft robotic actuator with fluidic network," IEEE Transactions on Robotics, vol. 34, no. 1, pp. 81-90, 2018.

[18] G. Runge and A. Raatz, "A framework for the automated design and modelling of soft robotic systems," CIRP Annals, vol. 66, no. 1, pp. 9-12, 2017.

[19] E. Coevoet, T. Morales-Bieze, F. Largilliere, Z. Zhang, M. Thieffry, M. Sanz-Lopez, B. Carrez, D. Marchal, O. Goury, J. Dequidt, and C. Duriez, "Software toolkit for modeling, simulation, and control of soft robots," Advanced Robotics, vol. 31, no. 22, pp. 1208-1224, 2017. [Online]. Available: https://doi.org/10.1080/01691864.2017.1395362

[20] C. Duriez, "Control of elastic soft robots based on real-time finite element method," in Robotics and Automation (ICRA), 2013 IEEE International Conference on. IEEE, 2013, pp. 3982-3987.
[21] Z. Zhang, J. Dequidt, A. Kruszewski, F. Largilliere, and C. Duriez, "Kinematic modeling and observer based control of soft robot using realtime finite element method," in 2016 IEEE/RSJ International Conference on Intelligent Robots and Systems (IROS), Oct 2016, pp. 5509-5514.

[22] T. George Thuruthel, Y. Ansari, E. Falotico, and C. Laschi, "Control strategies for soft robotic manipulators: A survey," Soft robotics, 2018.

[23] H. Jiang, Z. Wang, X. Liu, X. Chen, Y. Jin, X. You, and X. Chen, "A two-level approach for solving the inverse kinematics of an extensible soft arm considering viscoelastic behavior," in Robotics and Automation (ICRA), 2017 IEEE International Conference on. IEEE, 2017, pp. $6127-6133$

[24] T. G. Thuruthel, E. Falotico, F. Renda, and C. Laschi, "Learning dynamic models for open loop predictive control of soft robotic manipulators," Bioinspiration \& biomimetics, vol. 12, no. 6, p. 066003, 2017.

[25] K.-H. Lee, D. K. Fu, M. C. Leong, M. Chow, H.-C. Fu, K. Althoefer, K. Y. Sze, C.-K. Yeung, and K.-W. Kwok, "Nonparametric online learning control for soft continuum robot: An enabling technique for effective endoscopic navigation," Soft robotics, vol. 4, no. 4, pp. 324 337, 2017.

[26] M. Thieffry, A. Kruszewski, T.-M. Guerra, and C. Duriez, "Reduced Order Control of Soft Robots with Guaranteed Stability," in European Control Conference ECC18, Limassol, Cyprus, Jun. 2018.

[27] P. Benner, A. Cohen, M. Ohlberger, and K. Willcox, Model Reduction and Approximation: Theory and Algorithms. SIAM, 2017, vol. 15.

[28] J. Chenevier, D. González, J. V. Aguado, F. Chinesta, and E. Cueto, "Reduced-order modeling of soft robots," PloS one, vol. 13, no. 2, p. e0192052, 2018.

[29] S. H. Sadati, S. E. Naghibi, I. D. Walker, K. Althoefer, and T. Nanayakkara, "Control space reduction and real-time accurate modeling of continuum manipulators using ritz and ritz-galerkin methods," IEEE Robotics and Automation Letters, 2018.

[30] C. Della Santina, M. Bianchi, G. Grioli, F. Angelini, M. Catalano, M. Garabini, and A. Bicchi, "Controlling soft robots: balancing feedback and feedforward elements," IEEE Robotics \& Automation Magazine, vol. 24, no. 3, pp. 75-83, 2017.

[31] A. C. Antoulas, Approximation of large-scale dynamical systems. Siam, 2005, vol. 6.

[32] D. Ichalal, B. Marx, J. Ragot, and D. Maquin, "Simultaneous state and unknown inputs estimation with pi and pmi observers for takagi sugeno model with unmeasurable premise variables," in Control and Automation, 2009. MED'09. 17th Mediterranean Conference on. IEEE, 2009, pp. 353-358. 